\title{
Resultados clínicos y funcionales en reconstrucciones con aloinjertos intercalares de tibia
}

\author{
LUCAS LÓPEZ MILLÁN, LUIS APONTE TINAO, GERMÁN L. FARFALLI, \\ MIGUEL A. AYERZA y D. LUIS MUSCOLO \\ Instituto de Ortopedia y Traumatología "Carlos E. Ottolenghi" \\ Hospital Italiano de Buenos Aires
}

\begin{abstract}
RESUMEN
Introducción: El objetivo de este trabajo fue evaluar la supervivencia, las complicaciones y los resultados funcionales en un grupo de pacientes tratados con un aloinjerto intercalar de tibia luego de la resección tumoral primaria.
\end{abstract}

Materiales y métodos: Se evaluó a 26 pacientes tratados con aloinjertos intercalares de tibia luego de un defecto óseo secundario a una resección tumoral primaria. El seguimiento promedio fue de 6 años. La supervivencia del aloinjerto se determinó con el método de KaplanMeier. La evaluación funcional se realizó con la escala de la Musculoskeletal Tumor Society (MSTS). También, se registraron las complicaciones.

Resultados: La tasa de supervivencia global de las reconstrucciones fue del 84\% (IC 95\%: 90\%-70\%) a los 5 años y del $79 \%$ a los 10 años (IC 95\%: 95\%-63\%). Fue necesario remover el aloinjerto en 5 pacientes ( 3 casos por infección y 2 casos por recurrencia local). Dos pacientes presentaron falta de consolidación diafisaria y 3 sufrieron una fractura incompleta. En ninguno de estos casos, el aloinjerto debió ser removido. El resultado funcional promedio fue de 27 puntos, con un rango de 27 a 30 puntos. Esto corresponde a un miembro funcional con capacidad funcional aceptable y sin dolor limitante.

Conclusiones: A pesar de la incidencia de complicaciones, este análisis reveló una supervivencia aceptable con excelentes resultados funcionales. La reconstrucción con

Recibido el 9-10-2012. Aceptado luego de la evaluación el 19-10-2012. Correspondencia:

Dr. LUCAS LÓPEZ MILLÁN

lucas.lopez@hospitalitaliano.org.ar aloinjerto intercalar de tibia permite restaurar la anatomía de la pierna, con buenos resultados clínicos y funcionales.

\section{Palabras Clave: Aloinjerto intercalar. Tibia. \\ Clinical AND FunCtional OUTCOMES OF TIBIAL INTERCALARY ALLOGRAFTS RECONSTRUCTIONS}

\begin{abstract}
Background: The purpose of this study was to evaluate the survival, the complications and the functional outcome of intercalary tibial allografts reconstructions following tumor resections.

Methods: Intercalary tibia segmental allografts were implanted in 26 consecutive patients after segmental resections. Average follow-up was 6 years. Allograft survival was determined with the Kaplan-Meier method. Function was evaluated with the Musculoskeletal Tumor Society scoring system (MSTS).

Results: The rate of survival was $84 \%$ (CI 95\%: $90 \%$ $70 \%$ ) at 5 years and $79 \%$ at 10 years (CI 95\%: 95\%-63\%). Allografts were removed in 5 patients ( 3 due to infections and 2 due to local recurrences). Two patients showed diaphyseal nonunion and 3 had an incomplete fracture, but it was not necessary to remove the allografts. Average MSTS functional score was 29 points (range 27 to 30 ).

Conclusions: Despite the incidence of complications, this analysis showed an acceptable survival with excellent functional scores. The use of intercalary allograft clearly has a place in the reconstruction of a segmental defect created by the resection of a tumor in the diaphyseal and/or metaphyseal portion of the tibia.
\end{abstract}

KEY WORDS: Intercalary allografts. Tibia. 


\section{Introducción}

La tibia es un sitio común de aparición de tumores óseos primarios. ${ }^{1,2}$ Hay diversos abordajes terapéuticos indicados para tratar esta patología en esa localización, pero como está demostrado en la bibliografía, la combinación de quimioterapia sistémica con la resección quirúrgica del tumor con márgenes libres es la alternativa que ofrece mejores resultados. ${ }^{2,3}$ Este tratamiento permite conservar el miembro en un alto porcentaje de los pacientes generando, en definitiva, una pérdida esquelética masiva. Ante esta situación, existen diversas técnicas de reconstrucción, como los reemplazos protésicos ${ }^{4-7} \mathrm{o}$ las reconstrucciones biológicas, tales como la rotacionplastia, ${ }^{5,8}$ peroné autólogo microvascularizado asociado o no con aloinjertos, ${ }^{9-12}$ colocación solo de aloinjertos ${ }^{13}$ y otras técnicas, como las resecciones con distracción epifisaria descritas por Canadell, Forriol, Cara ${ }^{14}$ y SanJulian. ${ }^{15,16}$

A pesar de todas estas alternativas reconstructivas, se han publicado escasas comunicaciones que analicen exclusivamente una serie consecutiva de reconstrucciones segmentarias intercalares de tibia luego de la resección tumoral primaria. Por tal motivo, el objetivo de nuestro trabajo fue evaluar la supervivencia, las complicaciones y los resultados funcionales en un grupo de pacientes tratados con un aloinjerto intercalar de tibia.

\section{Materiales y métodos}

Entre mayo de 1991 y julio de 2008, se realizaron un total de 26 aloinjertos óseos intercalares de tibia por un defecto óseo masivo segmentario debido a una resección tumoral. Los pacientes debían tener las dos articulaciones adyacentes, tobillo y rodilla libres de movilidad. Todos los pacientes fueron sometidos a un seguimiento clínico y radiográfico mínimo de 2 años o hasta la falla del aloinjerto.

Se excluyó a todos los pacientes reconstruidos con trasplante intercalar de tibia, pero con artrodesis de rodilla, y aquellos con imposibilidad de completar el seguimiento mínimo de dos años.

Ningún paciente se perdió en el seguimiento en los primeros 2 años posteriores a la cirugía. El seguimiento promedio fue de 73 meses (rango de 9 a 176 meses). El diagnóstico inicial fue osteosarcoma (13 pacientes), fibrosarcoma (3 pacientes), condrosarcoma (2 pacientes), sarcoma de Ewing (1 caso), metástasis ósea (1 caso), tumor de células gigantes (1 caso), osteoblastoma ( 1 caso), fibroma condromixoide (1 caso), adamantinoma (1 caso), secuela de infección (1 caso) y fibrohistiocitoma maligno (1 caso). Dieciséis pacientes fueron sometidos a quimioterapia preoperatoria y los 10 restantes no recibieron ningún tratamiento adyuvante prequirúrgico. El promedio de edad era de 25 años (rango de 4 a 57 años). Trece pacientes eran del sexo femenino y 13 del sexo masculino.

Los aloinjertos no irradiados fueron recolectados de donantes cadavéricos y conservados de forma fresco congelado a una temperatura de $-80^{\circ} \mathrm{C} .{ }^{3} \mathrm{El}$ injerto fue seleccionado del banco de huesos, teniendo en cuenta el diámetro de la diáfisis tibial, sobre

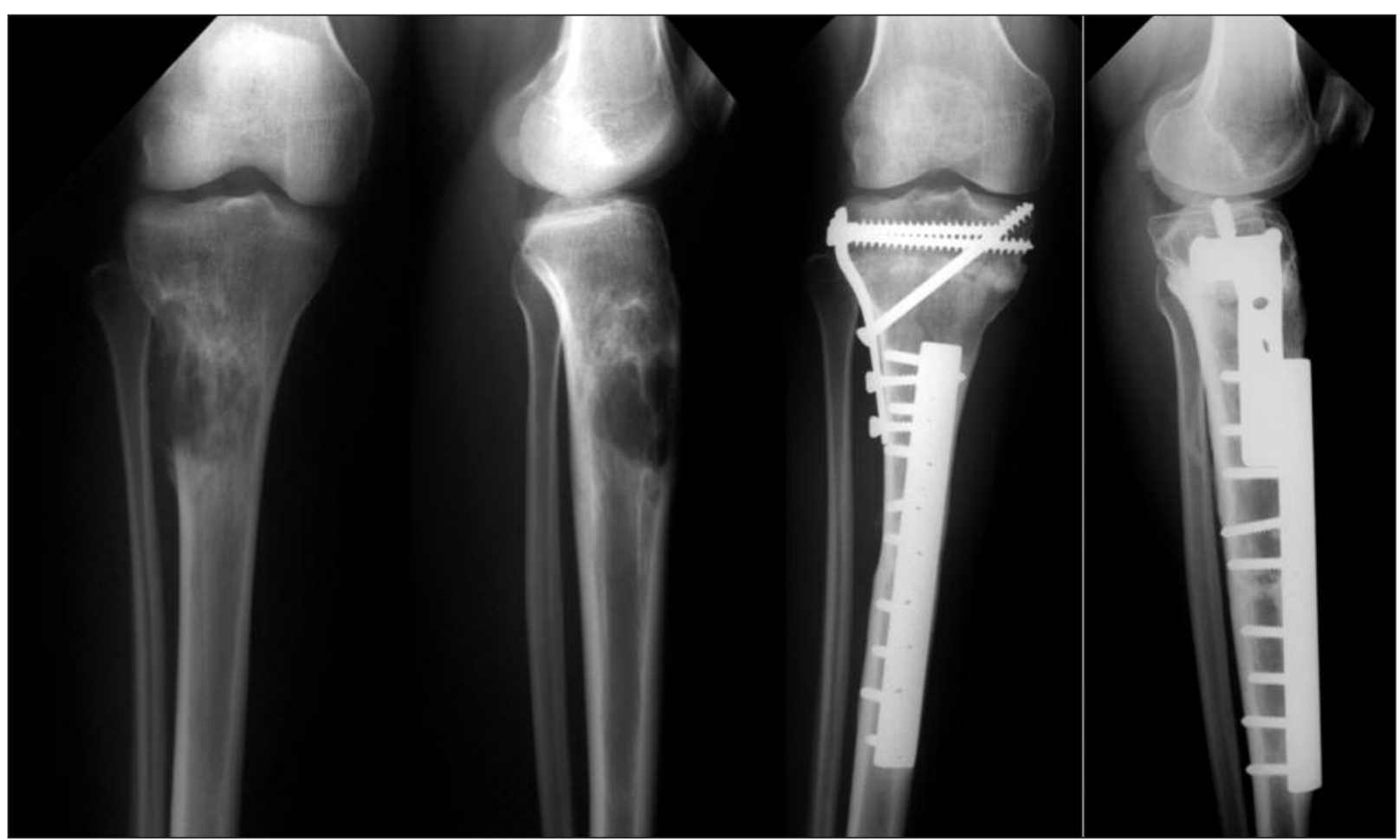

Figura 1. Ejemplo de trasplante intercalar estabilizado con placas y tornillos. 
la base de comparaciones radiográficas y tomográficas entre el hueso donante y el del receptor, para lograr la congruencia más anatómica posible.

La técnica quirúrgica comienza con la resección de la lesión ósea incluyendo la cicatriz de la biopsia con márgenes apropiados de hueso y de tejidos de partes blandas circundantes al tumor. A continuación, se prepara un injerto óseo fresco congelado, tallado previamente, a fin de suplantar con el mismo tamaño el segmento de hueso remanente. Luego, se talla el trasplante según la longitud del defecto por reconstruir, y se lo coloca en el defecto fijándolo básicamente con dos tipos de osteosíntesis: clavos endomedulares o con placa y tornillos (Fig. 1). Por último, se realiza un colgajo de gemelo cuando la reconstrucción involucra el tercio proximal de la tibia, para poder brindar mejor cobertura de las partes blandas y proteger el aparato extensor. El protocolo habitual de antibioticoterapia profiláctica incluyó cefazolina $1 \mathrm{~g}$, por vía endovenosa, en la inducción anestésica y por 48 horas después de la cirugía. Se utilizaron férulas en extensión de rodilla que se pueden quitar para practicar los ejercicios correspondientes. En años recientes, a la semana del posoperatorio, comenzamos a indicar ejercicios pasivos progresivos. En la mayoría de los pacientes, el seguimiento posoperatorio se realizó a la semana, a las dos semanas, al primero, segundo y tercer mes, y cada tres meses hasta completar el año y, luego, una vez al año. El par radiográfico se realizó en cada control a partir del mes del posoperatorio.

La supervivencia de los aloinjertos fue evaluada con el método de Kaplan-Meier. Se definió como falla cuando el aloinjerto debió ser removido, ya sea por revisión de la reconstrucción o por amputación del miembro.
Los pacientes fueron evaluados funcionalmente con la escala de la Musculoskeletal Tumor Society (MSTS). ${ }^{17}$ Este método de evaluación utiliza una escala de 0 a 5 puntos para cada criterio, y se le otorga un valor proporcional tomando 30 puntos como valor máximo.

Se empleó el análisis estadístico de regresión de Cox para evaluar los diferentes factores que influyeron sobre los resultados negativos de las reconstrucciones. Se consideró significativo a un valor $p<0,05$. El análisis estadístico se llevó a cabo con el programa SPSS 15.0 para Windows.

\section{Resultados}

La supervivencia global de los 26 trasplantes intercalares, calculada según el método de Kaplan-Meier, fue del 84\% (IC 95\%: 90\%-70\%) a los 5 años y del 79\% a los 10 años (IC 95\%: 95\%-63\%) y la mayoría de las fallas ocurrieron en los primeros 5 años (Fig. 2).

De las 26 reconstrucciones, 5 aloinjertos debieron ser removidos y fueron considerados como fallas. Las causas de estas fallas fueron 3 infecciones y 2 recurrencias locales que fueron tratadas con amputación. Además, se detectaron 2 casos de seudoartrosis diafisarias y 3 fracturas no desplazadas que se trataron colocando un autoinjerto y una nueva osteosíntesis (sin perder la reconstrucción original). Se observó una tendencia estadística a que los pacientes con quimioterapia puedan sufrir más com-

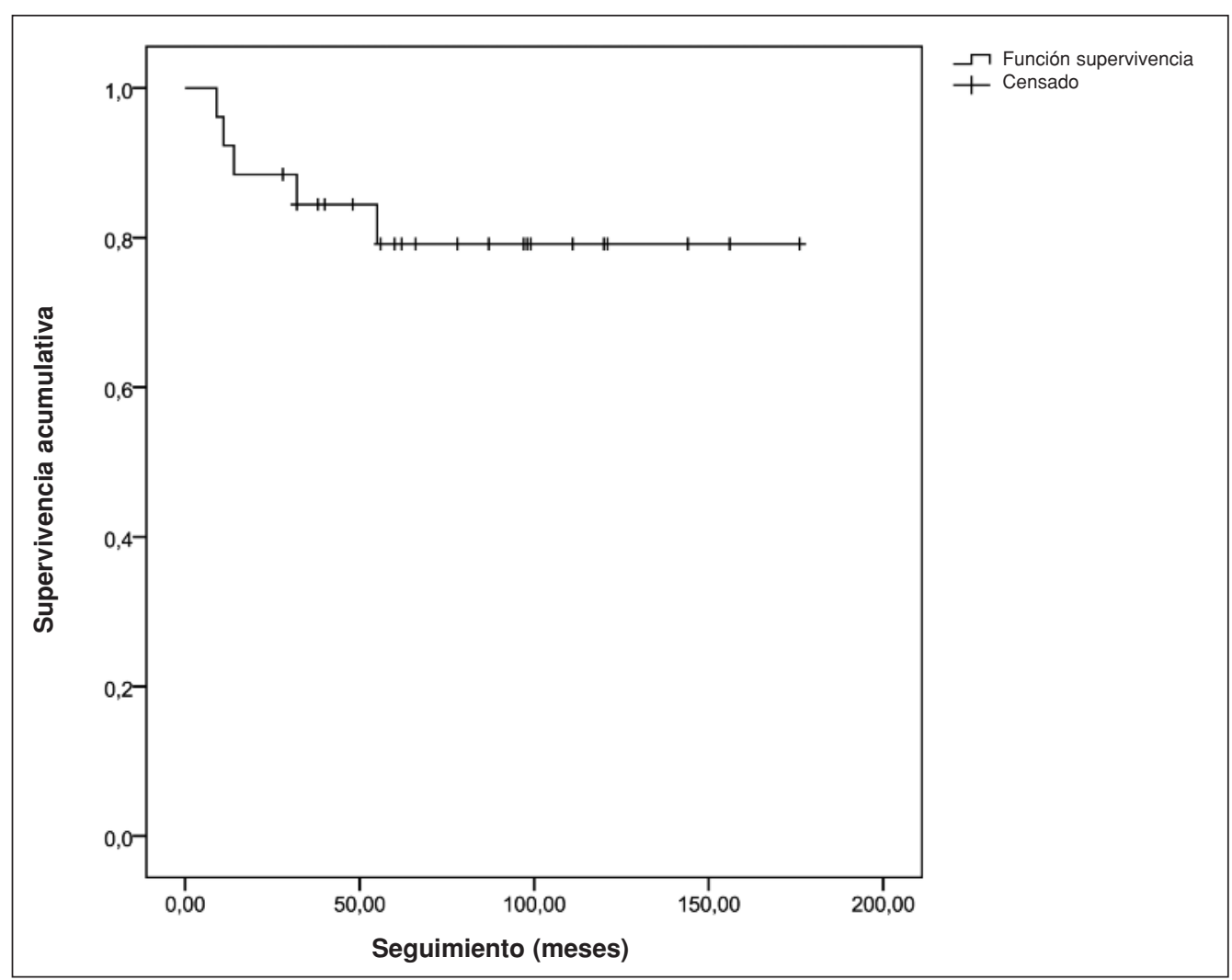

Figura 2. Gráfico que muestra la curva de supervivencia a los 5 y 10 años de seguimiento. 
plicaciones de cualquier tipo (con falla del trasplante o sin ella), pero esta tendencia no fue estadísticamente significativa $(p=0,07)$. No se observó que otros factores estén relacionados con un aumento significativo de falla específica del trasplante $(p>0,05)$.

El resultado funcional (MSTS) promedio de los pacientes que no tuvieron fallas fue de 27 puntos, con un rango de 27 a 30 puntos. Esto corresponde a un miembro funcional con muy buena capacidad funcional y sin dolor limitante.

\section{Discusión}

Los defectos segmentarios de la tibia pueden ser reconstruidos con distintas técnicas, que incluyen métodos biológicos o protésicos. ${ }^{5,9,10,15,16,18-24}$ Entre los métodos biológicos, el injerto autólogo, como el peroné vascularizado (asociado o no a un aloinjerto), puede tener una rápida incorporación, pero su longitud es limitada y puede alterar la longitud del miembro. También, hay que sumar al procedimiento secuelas en la zona donante. ${ }^{11,12}$

La distracción osteogénica es una manera fisiológica de reconstruir un segmento diafisario; sin embargo, las distracciones $>7$ u $8 \mathrm{~cm}$ no están exentas de complicaciones mayores. Además, ante resecciones extensas con epífisis remanentes pequeñas, su indicación es técnicamente cuestionable. ${ }^{15,16,23}$

Los aloinjertos intercalares impresionan como una alternativa válida para reconstruir este tipo de defectos diafisarios, con una supervivencia que supera el $70 \%$ a los 5 años y con resultados funcionales buenos y excelentes, ${ }^{17,21-23}$ comparables con nuestra serie.

La fijación de los implantes metálicos demanda la utilización de vástagos endomedulares que pueden ser o no cementados, pero requieren de una longitud determinada del canal medular remanente para poder tener una fijación estable y duradera. Además, surgen complicaciones mecánicas a largo plazo secundarias a aflojamiento. ${ }^{13}$ Observamos limitaciones evidentes en el diseño de nuestro trabajo. Al ser un estudio retrospectivo, hay variables no controladas difíciles de cuantificar, como la cantidad de partes blandas resecadas al extraer el tumor, la cantidad de compresión obtenida en la osteotomía donantereceptor, la osteosíntesis utilizada o la congruencia obtenida en las osteotomías donante-receptor. Asimismo, destacamos que el bajo número de pacientes que integran esta serie no permite realizar análisis estadísticos con resultados que demuestren intervalos de confianza con poder suficiente. Pero, a pesar de estas limitaciones, en la bibliografía, se publicaron escasos reportes con una serie consecutiva, un seguimiento a largo plazo y un número de casos similar al descrito.

Las fallas descritas de los aloinjertos son secundarias a recurrencias locales, infecciones, fracturas y seudoartrosis. ${ }^{5,14,16,19,24-30}$ En nuestra serie, la falla más frecuente fue la infección. Si se analiza la bibliografía, el índice de infección de aloinjertos intercalares varía del 5\% al $30 \% \cdot{ }^{17,20,31}$ En nuestra serie, la tasa de infección fue del $11 \%$. Consideramos que este porcentaje está dentro de lo esperable en este tipo de cirugía, pero continúa siendo elevado. Esta tasa de infección puede estar relacionada con la amplia exposición quirúrgica y con la íntima relación que tiene la tibia con la piel, es decir la escasa cobertura de partes blandas.

\section{Conclusiones}

Como conclusiones finales, creemos que, pese a la incidencia de complicaciones, este análisis reveló una supervivencia aceptable con excelentes resultados funcionales. La reconstrucción con aloinjerto intercalar de tibia permite restaurar la anatomía de la pierna proporcionando buenos resultados clínicos y funcionales.

\section{Bibliografía}

1. Bielack S, Kempf-Bielack B, Schwenzer D, Birkfellner T, Delling G, Ewerbeck V, et al. Neoadjuvant therapy for localized osteosarcoma of extremities. Results from the Cooperative osteosarcoma study group COSS of 925 patients. Klin Padiatr 1999;211:260-70.

2. Paulussen M, Ahrens S, Dunst J, Winkelmann W, Exner GU, Kotz R, et al. Localized Ewing tumor of bone: final results of the cooperative Ewing's Sarcoma Study CESS 86. J Clin Oncol 2001;19:1818-29.

3. Hermanek P, Wittekind C. The pathologist and the residual tumor (R) classification. Pathol Res Pract 1994;190:115-23.

4. Ham SJ, Schraffordt Koops H, Veth RP, Horn JR, Molenaar WM, Hoekstra HJ. Limb salvage surgery for primary bone sarcoma of the lower extremities: long-term consequences of endoprosthetic reconstructions. Ann Surg Oncol 1998;5:423-36.

5. Hillmann A, Hoffmann C, Gosheger G, Krakau H, Winkelmann W. Malignant tumor of the distal part of the femur or the proximal part of the tibia: endoprosthetic replacement or rotationplasty: functional outcome and quality-of-life measurements. $J$ Bone Joint Surg Am 1999;81(4):462-8.

6. Neel MD, Wilkins RM, Rao BN, Kelly CM. Early multicenter experienced with a noninvasive expandable prosthesis. Clin Orthop 2003;415:72-81. 
7. Unwin PS, Walker PS. Extendible endoprostheses for the skeletally immature. Clin Orthop 1996;322:179-93.

8. Hardes J, Gebert C, Hilmann A, Winkelmann W, Gosheger G. Rotationplasty in the surgical treatment plan of primary malignant bone tumors: possibilities and limits. Orthopade 2003;32:965-70.

9. Exner GU, Hauser R. Reconstruction of large segmental defects of the lower extremities following resection of malignant and locally aggressive bone tumors. Schweiz Rundsch Med Prax 1991;44:1214-24.

10. Hennen J, Sabo D, Martini AK, Bernd L. Mantel transplant for defect reconstruction after resection of malignant bone tumors of the lower extremity. Unfallchirurg 2002;105:120-7.

11. Manfrini M, Gasbarrini A, Malaguti C, Ceruso M, Innocenti M, Bini S, et al. Intraepiphyseal resection of the proximal tibia and its impact on lower limb growth. Clin Orthop Relat Res 1999;358:111-9.

12. Ozaki T, Hillmann A, Wuisman P, Winkelmann W. Reconstruction of tibia by ipsilateral vascularized fibula and allograft: 12 cases with malignant bone tumors. Acta Orthop Scand 1997;68:298-301.

13. Muscolo DL, Ayerza MA, Aponte-Tinao L, Ranalletta M, Abalo E. Intercalary femur and tibia segmental allografts provide an acceptable alternative in reconstructing tumor resections. Clin Orthop 2004;426:97-102.

14. Donati D, Capanna R, Campanacci D, Del Ben M, Ercolani C, Masetti C, et al. The use of massive bone allografts for intercalary reconstruction and arthrodeses after tumor resection: A multicentric European study. Chir Organi Mov 1993;78:8194.

15. San-Julian M, Aquerreta JD, Benito A, Canadell J. Indications for epiphyseal preservation in metaphyseal malignant bone tumors of children: relationship between image methods and histological findings. J Pediatr Orthop 1999;19:543-8.

16. San-Julian M, Dölz R, Garcia-Barrecheguren E, Noain E, Sierrasesumaga L, Cañadell J, et al. Limb salvage in bone sarcomas in patients younger than age 10: a 20-year experience. J Pediatr Orthop 2003;23:753-62.

17. Enneking WF, Dunham W, Gebhardt MC, Malawar M, Pritchard DJ. A system for the functional evaluation of reconstructive procedures after surgical treatment of tumors of the musculoskeletal system. Clin Orthop Relat Res 1993;286:241-46.

18. Abudu A, Carter SR, Grimer RJ. The outcome and functional results of diaphyseal endoprostheses after tumour excision. $J$ Bone Joint Surg Br 1996;78(4):652-7.

19. Cara JA, Laclériga A, Cañadell J. Intercalary bone allografts: 23 tumor cases followed for 3 years. Acta Orthop Scand 1994;65:42-6.

20. Gebhardt MC, Flugstad DI, Springfield DS, Mankin HJ. The use of bone allografts for limb salvage in high-grade extremity osteosarcoma. Clin Orthop Relat Res 1991;270:181-96.

21. Makley JT. The use of allografts to reconstruct intercalary defects of long bones. Clin Orthop Relat Res 1985;197:58-75.

22. Ortiz-Cruz EJ, Gebhardt MC, Jennings LC, Springfield DS, Mankin HJ. The results of transplantation of intercalary allografts after resection of tumors: A long-term follow-up study. J Bone Joint Surg Am 1997;79:97-106.

23. Canadell J, Forriol F, Cara JA. Removal of metaphyseal bone tumours with preservation of the epiphysis: physeal distraction before excision. J Bone Joint Surg Br 1994;76:127-32.

24. Berrey BH, Lord CF, Gebhardt MC, Mankin HJ. Fractures of allograft: Frequency, treatment, and end-results. J Bone Joint Surg Am 1990;72:825-33.

25. Gitelis S, Heligman D, Quill G, Piasecki P. The use of large allografts for tumor reconstruction and salvage of the failed total hip arthroplasty. Clin Orthop Relat Res 1988;231:62-70.

26. Hornicek FJ, Gebhardt MC, Tomford WW, Sorger JI, Zavatta M, Menzner JP, Mankin HJ. Factors affecting nonunion of the allograft-host junction. Clin Orthop Relat Res 2001;382:87-98.

27. Mankin HG, Springfield DS, Gebhardt MC, Tomford WW. Current status of allografting for bone tumors. Orthopedics 1992;15:1147-54.

28. Thompson RC, Pickvance EA, Garry D. Fractures in large segment allografts. J Bone Joint Surg Am 1993;75(11):1663-73.

29. Mnaymneh W, Malinin TI, Lackman RD, Hornicek FJ, Ghandur-Mnaymneh L. Massive distal femoral osteoarticular allografts after resection of bone tumors. Clin Orthop Relat Res 1994;303:103-115.

30. Vander Griend RA. The effect of internal fixation on the healing of large allografts. J Bone Joint Surg Am 1994;76(5):657-63.

31. Mankin HG, Gebhardt MC, Jennings LC, Springfield DS, Tomford WW. Long term results of allografts replacement in the management of bone tumors. Clin Orthop 1996;324:86-97. 